\title{
Avaliação da qualidade nutricional de composto orgânico produzido com resíduos de fumo
}

Dário C. Primo ${ }^{1}$, Francisco de S. Fadigas ${ }^{2}$, José C. R. Carvalho², Carlos D. S. Schmidt ${ }^{3} \&$ Antonio C. S. Borges Filho ${ }^{4}$

RESUMO

0 aproveitamento de resíduos agroindustriais na forma de composto orgânico, pode resultar em maior sustentabilidade para sistemas agrícolas. Propôs-se, neste trabal ho, analisar a qualidade final do composto orgânico em relação à composição em nutrientes e à presença de substâncias toxocologicamente ativas. Avaliaram-se as combinações de talo de fumo triturado, esterco e rúmen bovino ( $T F+E B+R B$ ), talos de fumo, esterco bovino e Microsept-Pó (TF+EB+ $M P$ ) e talos de fumo mais esterco bovino (TF+ EB). Amostras médias de cada composto foram analisadas para determinação da concentração em macro $\mathrm{e}$ micronutrientes, aos 60 e 120 dias e, para verificar a concentração de nicotina e a presença ou não de resíduos agrotóxicos na matéria-prima (TF) nos compostos obtidos aos 120 dias. Os dados obtidos demonstraram alta concentração de potássio (K), nitrogênio $(\mathrm{N})$, cloreto $(\mathrm{Cl})$ e ferro $(\mathrm{Fe})$ no composto final em relação aos demais macro e micronutrientes. $\mathrm{N}$ ão se detectaram resíduos de agrotóxicos na matéria-prima (TF) nem a presença de nicotina nas amostras obtidas aos 120 dias, demonstrando ausência de risco de impacto ambiental no uso agrícola desses compostos. No final do processo de compostagem a mistura TF+EB foi a que resultou no composto orgânico com maior concentração em macro e micronutrientes.

Palavras-chave: análise química, potencial nutricional, Nicotiana tabacum L

\section{Evaluation of the nutritional quality of organic compost produced with tobacco residues}

\begin{abstract}
The use of agroindustrial waste to produce organic compost can result in greater sustainability for agricultural systems. This study aimed to analyze the final quality of the organic compost, according to their nutrients and the presence of active toxic substances. The combinations of ground tobacco stem, bovine manure and rumen ( $T F+E B+R B$ ), tobacco stem, cattle manure and Microsept Dust ( $T F+E B+M P$ ) and tobacco stem and cattle manure ( $T F+E B$ ) were evaluated. Mean samples of each compost were analyzed to determine the concentration of micro and macro nutrients at 60 and 120 days and to verify the nicotine concentration and the presence or absence of pesticide residues in the raw material (TF) and in the composts obtained at 120 days. The data obtained showed high concentrations of potassium $(\mathrm{K})$, nitrogen $(\mathrm{N})$, chloride $(\mathrm{Cl})$ and iron $(\mathrm{Fe})$ in the final compost compared to the other micro and macro nutrients. Neither pesticides in the raw material (TF), nor presence of nicotine in the mean samples obtained within 120 days was detected, demonstrating the absence of environmental impact risks in the agricultural use of these composts. At the end of the composting process, the mixture TF+EB was the one which resulted in an organic compost with the highest concentration in micro and macro nutrients.
\end{abstract}

Key words: chemical analysis, nutritional potential, N icotiana tabacum L

\footnotetext{
1 UFRB. Rua da Mangueria, 51, Pera. CEP 48970-000, Senhor do Bonfim, BA. Fone: (74) 3541-3320. E-mail: darioprimo@gmail.com ${ }^{2}$ CETEC/U FRB, Campus Universitário de Cruz das Almas. CEP 44380-000. Cruz das Almas, BA. Fone: (75) 3621-3260. E-mail: fadigas@ufrb.edu.br ${ }^{2}$ CCAAB/UFRB Fone: (75) 3621-1220. E-mail: Jcrc48@yahoo.com.br

3 U FRB. Rua Embrapa, 732, Bairro Chapadinha. CEP 44380-000. Cruz das Almas, BA. Fone: (75) 8107-0609. E-mail: danieldanco@gmail.com ${ }^{4}$ UFRB. Rua E, 216 B, Inocoop, CEP 44380-000 Cruz das Almas, BA, Fone: (75) 9973-0871. E-mail: acsbf@ hotmail.com
} 


\section{INTRODUÇÃO}

O aproveitamento agrícola de resíduos agroindustriais na forma de composto orgânico, pode resultar em maior sustentabilidade dos sistemas agrícolas devido sobretudo ao fato de possibilitar a reciclagem de nutrientes no sistema e a redução da contaminação ambiental, decorrente de uma disposição inadequada. Os adubos orgânicos são produtos de cor escura, consistência friável, ricos em húmus, originados da ação fermentativa e digestiva de micro-organismos e de pequenos animais, em ambiente aeróbico, sobre matéria morta vegetal.

De acordo com a CEFS (1989) do estado da Bahia, os adubos orgânicos abrangem restos vegetais, resíduo de beneficiamento, adubos verdes, resíduos de animais, como esterco e resíduo de frigoríficos. Os adubos orgânicos possuem um conjunto de propriedades e características diferentes dos adubos minerais; na maior parte, nitrogênio, enxofre e boro são os nutrientes mais importantes (Raij, 1991).

A riqueza de um adubo orgânico em nutrientes depende do material de origem e do processo de produção. Os adubos orgânicos, além de fornecerem nutrientes se destacam por seu significativo papel, isto é, pelo fornecimento de matéria orgânica visando melhorar as propriedades físicas e biológicas do solo. Neste caso, o efeito é o de condicionador do solo considerando-se a matéria orgânica um produto que melhora os atributos físicos do solo (Raij, 1991).

Dada à característica dos compostos orgânicos de reterem nutrientes e liberá-los para a solução do solo na função da sua decomposição, os mesmos podem contribuir com a diminuição no número de parcelamentos da adubação mineral reduzindo os gastos com mão-de-obra e garantindo melhor eficiência da adubação. A prática de adubação orgânica anual em solos intemperizados de ecossistemas tropicais tem contribuído pouco com o aumento do estoque de carbono orgânico no solo. Contudo, solos constituídos de argilominerais 2:1, como os do nordeste brasileiro, apresentam capacidade de reter o carbono aplicado na forma de adubo orgânico, considerados sumidouros desse elemento (Malavolta, 1980).

Os elementos essenciais às plantas são divididos em dois grandes grupos, dependendo das quantidades exigidas pelas plantas: macronutrientes $\mathrm{N}, \mathrm{P}, \mathrm{K}, \mathrm{Ca}, \mathrm{Mg}$ e $\mathrm{S}$ e micronutrientes $\mathrm{B}, \mathrm{Cl}, \mathrm{Cu}, \mathrm{Fe}, \mathrm{Mn}, \mathrm{Mo}, \mathrm{Zn}$. A legislação brasileira de fertilizantes e corretivos, subdivide os macronutrientes em duas categorias: macronutrientes primários $\mathrm{N}, \mathrm{P}, \mathrm{K}$ e macronutrientes secundários, $\mathrm{Ca}, \mathrm{Mg}$ e $\mathrm{S}$. Em nutrição de plantas os teores de macronutrientes são, em geral, dados como \% da forma elementar dependendo, por vezes, da finalidade, aparecem como equivalente mg. Comumente, a concentração dos micronutrientes é fornecida em parte por milhão (ppm) a exemplo da liberação dada em $\mathrm{mg} \mathrm{kg}^{-1}$ (Malavolta, 1980).

A utilização de resíduos agroindustriais na forma de composto orgânico pode favorecer as propriedades físicas, químicas e biológicas do solo (Alcarde et al., 1989; Ros et al., 2001; Breson et al., 2001), visto que pode disponibilizar elementos necessários à nutrição da planta (Kiehl, 1998). Não obstante o aumento da CTC dos solos tropicais, decorrente da adubação orgânica, constitui importante mecanismo de melhoria da fertilidade desses solos além de favorecer a ciclagem microbiana de nutrientes (Sanchez, 1997).

Os resíduos gerados nas atividades agroindustriais sejam eles sólidos ou líquidos possuem, em geral, grande potencial agrícola em virtude de serem ricos em matéria orgânica e nutrientes utilizados pelas plantas e micro-organismos do solo. A utilização de composto na atividade agronômica depende sobretudo da sua qualidade, especialmente do conteúdo em matéria orgânica da sua maturidade, da concentração em nutrientes e da presença ou ausência de substâncias potencialmente perigosas e indesejáveis ao ambiente agrário (Zucconi \& Bertoldi 1981, Bertoldi \& Griselli 1992). Objetivou-se, neste trabalho, analisar a qualidade final do composto orgânico produzido com resíduos da cultura do fumo em relação à composição em nutrientes e à presença de substâncias toxicologicamente ativas.

\section{MATERIAL E MÉTODOS}

O experimento para avaliação da qualidade nutricional do composto orgânico produzido com resíduo da cultura do fumo foi conduzido na Fazenda Capivari, de propriedade da empresa Danco, localizada no município de Governador Mangabeira, BA, de dezembro de 2007 a março de 2008.

Obtiveram-se as amostras para caracterização química a partir de seis pilhas de cada composto originando uma amostra média de cada tratamento. Avaliaram-se as combinações de talo de fumo $(85 \%)$, esterco $(15 \%)$ e rúmen bovino $(0,26 \%)$ $(\mathrm{TF}+\mathrm{EB}+\mathrm{RB})$; talo de fumo $(85 \%)$, esterco bovino $(15 \%)$ e microsept-pó $(0,13 \%)(\mathrm{TF}+\mathrm{EB}+\mathrm{MP})$ e talo de fumo $(85 \%)$ mais esterco bovino (15\%) (TF+EB).

Com vistas à caracterização da matéria-prima (talo de fumo + folhas novas + flores) coletaram-se amostras recém-picadas; tanto as amostras de TF picado como as dos diferentes tratamentos, foram secadas em estufa com ventilação forçada a $65^{\circ} \mathrm{C}$, durante $72 \mathrm{~h}$ e posteriormente trituradas em moinho de facas tipo Wiley com peneira de malha $1 \mathrm{~mm}$. As amostragens do composto foram realizadas no início do processo, após a montagem das pilhas e aos 60 e 120 dias de compostagem.

Cerca de $50 \mathrm{~g}$ de amostras trituradas de cada tratamento foram encaminhados ao Laboratório Unithal, em Campinas, São Paulo, para determinação da concentração de macro (N, $\mathrm{P}, \mathrm{K}, \mathrm{Ca}, \mathrm{Mg}$, e S) e micronutrientes ( $\mathrm{Fe}, \mathrm{Cl}, \mathrm{Cu}, \mathrm{Mn}, \mathrm{Zn}, \mathrm{B}$, e $\mathrm{Mo}$ ) sendo a concentração de $\mathrm{N}$ determinada pelo método de Kjeldahl, a de fósforo por colorimetria e a de $\mathrm{Ca}, \mathrm{Mg}, \mathrm{Cu}, \mathrm{Fe}$, Zn e Mn, por espectrofotometria de absorção atômica. A determinação de K foi realizada por fotometria de chama (Silva, 1999).

Realizaram-se, também, análises no Laboratório da Universidade de Santa Cruz (UNISC), no Rio Grande Sul, para determinação da concentração de nicotina, por espectrometria na matéria-prima (TF) e no composto obtido aos 120 dias. Para verificar a existência ou não de resíduos de defensivos agrícolas utilizados na cultura do fumo para controle de pragas e doenças na matéria-prima enviaram-se, ao Laboratório da Bioensaios, do Rio Grande Sul, amostras, em que foi feita uma análise de varredura para detecção dos princípios ativos pre- 
sentes em agrotóxicos cujas determinações foram realizadas por cromatografia gasosa e cromatografia líquida, acopladas a espectrômetro de massa.

\section{RESULTADOS E DISCUSSÃO}

Na matéria-prima, o potássio (K) foi o elemento que mais se destacou entre os macronutrientes, seguido do nitrogênio (N). Em relação aos micronutrientes, também na matéria-prima, o cloro $(\mathrm{Cl})$ e o ferro $(\mathrm{Fe})$ foram os que apresentaram maiores concentrações (Tabela 1). Os resultados encontrados em concentração de macronutrientes nas amostras médias obtidas durante o processo de compostagem (60 dias) e do composto final (120 dias), apresentaram pequena diferença em relação às concentrações encontradas na matéria-prima.

Tabela 1. Concentração de macro e micronutrientes na matériaprima (TF) secada a $650 \mathrm{C}$

\begin{tabular}{cccc}
\hline Macronutrientes Concentração (\%) & Micronutrientes & Concentração $\left(\mathbf{m g ~ k g}^{-1}\right)$ \\
N & 1,79 & $\mathrm{Fe}$ & 1450,0 \\
$\mathrm{P}$ & 0,15 & $\mathrm{Cl}$ & 8000,0 \\
$\mathrm{~K}$ & 2,33 & $\mathrm{Cu}$ & 135,0 \\
$\mathrm{Ca}$ & 0,40 & $\mathrm{Mn}$ & 45,0 \\
$\mathrm{Mg}$ & 0,26 & $\mathrm{Zn}$ & 43,0 \\
$\mathrm{~S}$ & 0,24 & $\mathrm{~B}$ & 16,8 \\
- & - & $\mathrm{Mo}$ & 3,0 \\
\hline
\end{tabular}

O potássio e o nitrogênio foram os elementos que apresentaram maior concentração, de forma similar ao encontrado na matéria-prima. A adição do inoculante esterco bovino pode ter sido o fator que favoreceu o aumento da concentração desses elementos no composto estabilizado e no composto final.

Comparando-se o teor de nitrogênio inicial do composto orgânico (Tabela 2) com o teor no final da compostagem, observa-se uma pequena redução no composto $\mathrm{TF}+\mathrm{EB}+\mathrm{RB}$ e $\mathrm{TF}+\mathrm{EB}$ devido, possivelmente, à ação da temperatura durante o processo da compostagem a qual tem grande influência na volatilização do nitrogênio na forma de amônia $\left(\mathrm{NH}_{4}\right)$, o $\mathrm{pH}$ do composto era alcalino e o meio continha bastante umidade (55 a 64\%). A elevação de temperatura a níveis próximos ou acima de $70^{\circ} \mathrm{C}$, segundo Kiehl (1998), pode promover perdas de nitrogênio amoniacal; neste sentido Prochnow

Tabela 2. Concentração de macronutrientes do composto estabilizado (60 dias) e no composto final (120 dias)

\begin{tabular}{ccccccc}
\hline \multirow{2}{*}{ Tratamentos } & $\mathbf{7}$ Macronutrientes (\%) \\
\cline { 2 - 7 } & $\mathbf{N}$ & $\mathbf{P}$ & $\mathbf{K}$ & $\mathbf{C a}$ & $\mathbf{M g}$ & $\mathbf{S}$ \\
TF+ EB+RB & 1,90 & 0,20 & 2,41 & 0,82 & 0,47 & 0,27 \\
TF+ EB+ MP & 1,60 & 0,20 & 2,28 & 0,78 & 0,47 & 0,32 \\
TF+ EB & 2,05 & 0,21 & 2,92 & 0,81 & 0,48 & 0,29 \\
\hline & & \multicolumn{7}{c}{ Composto final (120 dias) } \\
TF+ EB+ RB & 1,64 & 0,19 & 2,38 & 0,73 & 0,42 & 0,26 \\
TF+ EB+ MP & 1,67 & 0,19 & 2,28 & 0,80 & 0,45 & 0,32 \\
TF+ EB & 1,85 & 0,23 & 2,53 & 0,84 & 0,50 & 0,32 \\
\hline
\end{tabular}

et al. (1998), também citam, em seu trabalho, que a perda de amônia atingiu valores máximos ( $84 \%$ do total) nas primeiras três semanas, coincidindo com a fase de temperaturas elevadas.

Pagans et al. (2006), associaram as perdas de amônia ao aumento da temperatura na fase termofílica do processo cuja explicação estaria no fato de, na fase inicial do processo, ocorrer grande degradação de substâncias orgânicas facilmente biodegradáveis, com alto teor de $\mathrm{N}$, causando a liberação do gás de amônia, o qual é exponencialmente dependente da temperatura.

Em análises realizadas por pesquisadores da Embrapa Mandioca e Fruticultura Tropical (EMBRAPA, 2005), com resíduos de fumo secados a $110^{\circ} \mathrm{C}$, resultados semelhantes foram encontrados aos obtidos para a matéria-prima em que as percentagens de N, P e K são iguais a 2,17, 0,51 e 2,78, respectivamente.

Sediyama et al. (2000) encontraram, em trabalho realizado comparando composto orgânico oriundo de resíduo de Leucena com esterco animal quanto aos teores de N, P e K, valores em percentagem também similares; esterco de curral 2,60; 2,40 e 2,25; esterco de frango 2,$00 ; 2,00 ; 1,00$ e no composto orgânico curado 1,$59 ; 0,27 ; 0,98$.

A concentração de micronutrientes em mg kg-1, tanto durante o processo da compostagem (60 dias) quanto no composto obtido (120 dias) foi mais expressiva para o ferro, seguida do cloro total, ao contrário dos resultados obtidos na matéria-prima (Tabela 3). Segundo Malavolta (1980), o ferro é o micronutriente que se apresenta em maior teor no solo; nos solos brasileiros o conteúdo total varia entre 22 e $40 \%$ em mg $\mathrm{kg}^{-1}$.

Tabela 3. Concentração de micronutrientes do composto estabilizado (60 dias) e no composto final (120 dias)

\begin{tabular}{|c|c|c|c|c|c|c|c|c|}
\hline \multirow{2}{*}{ Tratamentos } & \multicolumn{8}{|c|}{ Micronutrientes $\left(\mathrm{mg} \mathrm{kg}^{-1}\right)$} \\
\hline & $\mathrm{Fe}$ & $\mathrm{Cl}$ & Mn & $\mathrm{Cu}$ & $\mathrm{Zn}$ & B & Mo & Co \\
\hline \multicolumn{9}{|c|}{ Composto estabilizado (60 dias) } \\
\hline $\mathrm{TF}+\mathrm{EB}+\mathrm{RB}$ & 6500,0 & 6200,0 & 210,0 & 100,0 & 65,0 & 20,8 & 5,0 & 4,0 \\
\hline$T F+E B+M P$ & 7000,0 & 4500,0 & 192,0 & 158,0 & 170,0 & 23,1 & 5,0 & 3,0 \\
\hline$T F+E B$ & 6800,0 & 4500,0 & 200,0 & 127,0 & 67,0 & 20,0 & 4,0 & 5,0 \\
\hline \multicolumn{9}{|c|}{ Composto final (120 dias) } \\
\hline$T F+E B+R B$ & 7800,0 & 6200,0 & 180,0 & 300,0 & 87,0 & 16,8 & 3,0 & 5,0 \\
\hline$T F+E B+M P$ & 7600,0 & 5300,0 & 195,0 & 350,0 & 100,0 & 20,0 & 4,0 & 4,0 \\
\hline$T F+E B$ & 8000,0 & 7000,0 & 230,0 & 270 & 100,0 & 20,0 & 4,0 & 5,0 \\
\hline
\end{tabular}

O molibdênio e o cobalto apresentaram menor concentração durante o processo da compostagem e no composto obtido; contudo, os resultados mostram que o composto obtido é de boa qualidade uma vez que contém todos os macros e micronutrientes em concentrações consideradas ideais para a agricultura (Malavolta, 1980).

Sabe-se que a folha do fumo contém a molécula orgânica da nicotina $\left(\mathrm{C}_{10} \mathrm{H}_{14} \mathrm{~N}_{2}\right)$ como princípio ativo, em que esta possui efeito antagônico sobre micro-organismo e insetos. Segundo Lovatto et al. (2004) a nicotina composto orgânico, principal alcalóide do tabaco, é utilizada como inseticida na agricultura. 
As análises realizadas indicam haver uma pequena concentração de nicotina na matéria-prima (TF) (Tabela 4), embora não tenha sido detectada em amostras compostas dos compostos obtidos aos 120 dias. Por se tratar de uma molécula orgânica acredita-se que, provavelmente, a molécula da nicotina tenha sido degradada durante o processo da compostagem.

Tabela 4. Análise química do teor de nicotina no material de origem e nos compostos obtidos aos 120 dias $^{1}$

\begin{tabular}{cc}
\hline Material & Resultados $\mathbf{~} \mathbf{~ 1 0 0 ~}^{\mathbf{1}}$ \\
Matéria Prima (TF) & 0,001 \\
TF+EB+RB & $<0,001$ \\
TF+EB+MP & $<0,001$ \\
TF+EB & $<0,001$ \\
\hline
\end{tabular}

${ }^{1}$ Amostras secas a $65{ }^{\circ} \mathrm{C}$

Esta degradação pode ter sido provocada não só pelo aumento de temperatura das pilhas, que alcançou valores em torno de $70{ }^{\circ} \mathrm{C}$ no início do processo e permaneceu entre 50 e $60^{\circ} \mathrm{C}$ até os 40 dias da compostagem, mas também pela ação de micro-organismos decompositores, de vez que são bastante ativos até a fase de estabilização. Ruan et al. (2005) afirmam que bactérias Pseudomonas sp. strain HF-1 têm forte habilidade para degradar a nicotina podendo ser utilizadas para biorremediação de ambientes contaminados por resíduos de tabaco. O aumento da temperatura no processo de compostagem até atingir o estado mesofílico ocorre poucos dias após a montagem da pilha; a seguir, a temperatura sobe até cerca de $60{ }^{\circ} \mathrm{C}$ dando-se o estádio termofílico no qual a decomposição é máxima e pode durar mais de 20 dias. A partir deste período a pilha vai diminuindo de tamanho e ficando mais escura, reflexo da decomposição das substâncias orgânicas, promovida pela atividade microbiana (Witter \& Lopez, 1987).

A temperatura é fator importante, sobretudo no que diz respeito a rapidez do processo de biodegradação e a eliminação de patógenos, sendo também um indicativo da ação biológica que reflete a eficiência do processo. Do ponto de vista aplicado, este resultado mostra o potencial para uso agrícola desse tipo de composto orgânico, vez que não se espera encontrar qualquer impacto ambiental decorrente da presença de nicotina no composto final.

No plantio do fumo, em que se coletou a matéria-prima para produção do composto orgânico, são utilizados os seguintes princípios ativos para o controle de pragas e doenças: Azoxystrobin (Estrobilurina), Lambdacyhalothrin (Piretróide), Deltamethrin (Piretróide), Acephate (Organofosforado), Tebuconazole (Triazol), Mancozeb (Ditiocarbamato) e Imidacloprid (Neonicotinóide). Desses grupos foram analisados os Organofosforados, os Piretróides e Ditiocarbamatos. Os resultados analíticos apresentados (Tabela 5) indicaram que não foi detectada a presença desses agrotóxicos na matéria-prima (TF) não sendo necessário, portanto, realizar análises no composto obtido aos 120 dias para a presença dessas substâncias. Não foi possível analisar a matéria-prima em relação aos demais grupos químicos.
Tabela 5. Resultados obtidos para a análise de detecção de resíduos de agrotóxicos na matéria-prima secada a $65^{\circ} \mathrm{C}$ (Laudo Analítico BQ -24348/08)

\begin{tabular}{cccc}
\hline \multicolumn{1}{c}{ Parâmetro } & Resultado $\left(\mathbf{m g ~ k g}^{-1}\right)$ & Metodologia & LOQ $^{\mathbf{~}} \mathbf{m g ~ k g}^{\mathbf{1}}$ ) \\
Dithiocarbamates (CS2) & $\mathrm{ND}^{1}$ & $\mathrm{GC} \mathrm{MS}^{3}$ & 0,01 \\
Organofosforados & $\mathrm{ND}$ & $\mathrm{LC} \mathrm{MS}$ & 0,01 \\
Piretróides & $\mathrm{ND}$ & $\mathrm{LC} \mathrm{MS}$ & 0,01 \\
\hline
\end{tabular}

${ }^{1} \mathrm{ND}=\mathrm{Não}$ detectado; ${ }^{2} \mathrm{LOQ}=$ Limite de quantificação;

${ }^{3} \mathrm{GC}$ MS=cromatografia gasosa acoplada a espectrômetro de massa ${ }^{4} \mathrm{LC}$ MS=cromatografia líquida acoplada a espectrômetro de massa

Este é outro aspecto positivo na obtenção de composto de resíduo de fumo que indica não haver restrições no uso em relação à possibilidade de causar impacto negativo no ambiente; assim, as análises dos resultados comprovam que o composto de talos de fumo pode ser utilizado para fins agrícolas.

\section{CONCLUSÕES}

1. Ao final do processo de compostagem, o tratamento em que foi adicionado apenas esterco bovino aos talos de fumo triturados, foi o que resultou em um composto orgânico com maior concentração em macro e micronutrientes.

2. O processo de compostagem foi eficiente em eliminar as moléculas de nicotina presentes no resíduo da cultura do fumo (talo, folhas novas e flores).

3. Os resultados analíticos não indicaram a presença de agrotóxicos na matéria- prima (Organofosforados, os Piretróides e Ditiocarbamatos), atestando a qualidade do resíduo para uso na produção de composto orgânico visando ao uso agrícola.

\section{LITERATURA CITADA}

Alcarde, J. C.; Guidolin, J. A.; Lopes, A. S. Os adubos e a eficiência das adubações. São Paulo: ANDA, 1989. 335p. Boletim Técnico.

Bertoldi, M. de.; Griselli, V. M. C. Microbial populations in the compost process In: Composting the staff of compost science and land utilization. New York: J. G. Press, Emmaus Pam, 1992. 26p.

Breson, L. M.; Koch, Y.; Bissonnais, L. E; Barriuso, E.; Lecomte, V. Soil surface stabilization by municipal waste compost application. Soil Science Society Journal of American v.65, p.1804-1811, 2001.

CEFS - Comissão Estadual de Fertilidade do Solo. Manual de adubação e calagem para o estado da Bahia. 2.ed. Salvador: CEPLAC/EMATERBA, EMBRAPA, EPABA/NITROFETIL, 1989. 176p.

EMBRAPA - Empresa Brasileira de Pesquisa Agropecuária. Compostagem caseira de lixo orgânico doméstico. Cruz das Almas: Embrapa CNPMFT, 2005. 12p. Circular técnica, 76.

Kiehl E. J. Fertilizantes orgânicos. São Paulo: Agronômica Ceres, 1998. 326p. 
Lovatto, P. B.; Goetze, M.; Thomé, G. S. H. Efeito de extratos de plantas silvestres da família solanaceae sobre o controle de brevicoryne brassicae em couve (Brassica oleracea var. acephala). Ciência. Rural, v.34, n.4, p.971-978, 2004.

Malavolta, E. Elementos de nutrição mineral de plantas. São Paulo: Agronômica Ceres, 1980. 228p.

Pagans, E.; Barrena, R.; Font, X.; Sánchez, A. Ammonia emissions from the composting of different organic wastes. Dependecy on process temperature. Chemosphere, v.62, p.1534-1542, 2006.

Prochnow, L. I.; Cunha, C. F. da.; Kiehl, J. E.; Alcarde, J. C. Controle da volatilização de amônia durante a compostagem, através da adição de superfosfato simples, superfosfato triplo e gesso agrícola com diferentes níveis de acidez residual. In: Fertbio, 1, 1998, Caxambu. Anais... Lavras: SBCS, 1998. 74p.

Raij, B. van. Fertilidade do solo e adubação. Piracicaba: Agronômica Ceres, 1991. 328p

Ros, M.; Garcia, C.; Hernandez, T. The use of urban wastes in the control of erosion in semiarid Mediterranean soil. Soil Use and Management, v.17, n.4, p.292-293, 2001.
Ruan, A.; Min, H.; Peng, X.; Zheng, H. Isolation and charatterization of Pseudomonas sp. Strain HF-1, capable of degrading nicotine. Research in Microbiology, v.156, p.700/7006, 2005.

Sanchez, P. A. Changing tropical soil fertility paradigms: From Brazil to Africa and back. In: Moniz, A. C.; Furlani, A. M. C.; Schaeffert, R. E.; Fageria, N.; Zucconi, F. F. M.; Bertoldi, M. Biological Evaluation of Compost Maturyty Biocycle, v.22, n.4, p.27-29, 1997.

Sediyama, M. A. N.; Garcia, N. C. P.; Vidigal, S. M.; Matos, A. T. de. Nutrientes em compostos orgânicos de resíduos vegetais e animais. Scientia Agrícola. v.57, n.1 p.128-135, 2000.

Silva, F. C. da. Manual de análises de solos, plantas e fertilizantes. Brasília: Embrapa Solos, 1999. 370p.

Witter, E.; Lopez, J. M. Monitoring the composting process using parameters of compost stability. In: de Bertoldi, M. P. Ferranti, M. P.; L'Hermite, P.; Zucconi, F.,(ed.). Compost: Quality and use. London: Elsevier Applied Science, 1987. p.351-358.

Zucconi, F. F. M.; Bertoldi, M. Biological evaluation of compost maturity Biocycle, v.22, n.4, p.27-29, 1981. 